\title{
Work plan for improving the DARWIN2.3 depleted material balance calculation of nuclides of interest for the fuel cycle
}

\author{
Axel Rizzo ${ }^{1, a}$, Claire Vaglio-Gaudard ${ }^{1}$, Julie-Fiona Martin ${ }^{2}$, Gilles Noguère ${ }^{1}$, and Romain Eschbach ${ }^{1}$ \\ 1 CEA, DEN, DER, SPRC, Cadarache, 13108 Saint-Paul-lez-Durance, France \\ 2 AREVA-NC, BU Recyclage - Paris, France
}

\begin{abstract}
DARWIN2.3 is the reference package used for fuel cycle applications in France. It solves the Boltzmann and Bateman equations in a coupling way, with the European JEFF-3.1.1 nuclear data library, to compute the fuel cycle values of interest. It includes both deterministic transport codes APOLLO2 (for light water reactors) and ERANOS2 (for fast reactors), and the DARWIN/PEPIN2 depletion code, each of them being developed by CEA/DEN with the support of its industrial partners.

The DARWIN2.3 package has been experimentally validated for pressurized and boiling water reactors, as well as for sodium fast reactors; this experimental validation relies on the analysis of post-irradiation experiments (PIE).

The DARWIN2.3 experimental validation work points out some isotopes for which the depleted concentration calculation can be improved. Some other nuclides have no available experimental validation, and their concentration calculation uncertainty is provided by the propagation of a priori nuclear data uncertainties.

This paper describes the work plan of studies initiated this year to improve the accuracy of the DARWIN2.3 depleted material balance calculation concerning some nuclides of interest for the fuel cycle.
\end{abstract}

\section{Introduction}

DARWIN2.3 [1] is the French reference package used for fuel cycle applications. It solves the Boltzmann and Bateman equations in a coupling way to compute fuel cycle parameters, at any irradiation and cooling time. The quantities of interest for fuel cycle are fuel inventory, masses, residual decay heat, neutron, $\alpha, \beta, \gamma$ sources and spectra, and radiotoxicity. A package is defined by a nuclear data library, one or several computer codes, and one or several calculation schemes (for instance: a reference routine and an industrial routine). For DARWIN2.3, nuclear data used comes from the JEFF3.1.1 evaluation [2].

DARWIN2.3 includes both deterministic transport codes APOLLO2 [3] (for light water reactors) and ERANOS2 [4] (for fast reactors), which provide neutronic data to the DARWIN/PEPIN2 depletion solver [5], each of them being developed by CEA/DEN with the support of its industrial partners. These data are self-shielded cross sections libraries and collapsed neutron fluxes.

In addition, data such as multigroup activation cross sections at infinite dilution, a full filiation chain, and specific nuclear constants are used in the DARWIN/PEPIN2 solver, directly taken from JEFF-3.1.1. This allows taking into account isotopes and reactions that are not described in the simplified filiation chain used in APOLLO2 or ERANOS2.

The reference calculation scheme used for PWR DARWIN2.3 calculations, called CYCLE2008-PWR, is

a e-mail: axel.rizzo@cea.fr based on the recommended APOLLO2.8 calculation scheme [6] used for neutron transport calculations. They mainly differ in the flux solver used. From the JEFF-3.1.1 multigroup nuclear data, a spatial selfshielding calculation is carried out with SHEM [7] recommendations. Then the neutron flux is calculated using a Pij flux solver to provide neutronic data for the first depletion step. Once the depletion calculation is achieved, the fuel inventory is updated, and so is the flux thanks to a Pij method-based calculation at specific burn up values. The self-shielded cross sections are also updated along the whole depletion calculation at specific burn up values. All these data are stored in a file that will be used by the DARWIN/PEPIN2 depletion solver.

The DARWIN2.3 package has been experimentally validated for light water reactors for the material balance and decay heat calculation [1]. It has also been experimentally validated for sodium fast reactors for the material balance of the main actinides and fission products involved in burn up-credit calculations [8]. This experimental validation relies on the analysis of dedicated PIE, mostly consisting in the chemical characterization of in-pile irradiated fuel pellets.

The experimental validation of the DARWIN2.3 package for material balance calculation was performed for a large range of burnup from 10 to $85 \mathrm{GWd} / \mathrm{t}$ for UOX fuels and 10 to $60 \mathrm{GWd} / \mathrm{t}$ for MOX fuels. As regards decay heat calculation for PWR UOX fuel assemblies, the experimental validation of the package covers both short cooling time (40 minutes - 40 days) for lowburnup fuels [9] and long cooling time (10-30 years) for intermediate to high burnup (20-50 $\mathrm{GWd} / \mathrm{t})$ ranges [1]. 
Table 1. Results of the DARWIN2.3 experimental validation of some important isotopes in PWR UOX fuel.

\begin{tabular}{|l|c|c|}
\hline Isotope & $\begin{array}{c}\text { Mean }(\mathbf{C}-\mathrm{E}) / \mathbf{E} \text { value } \\
\text { tendency } \pm \text { standard } \\
\text { deviation at } \mathbf{1} \boldsymbol{\sigma}\end{array}$ & Related topics of study \\
\hline${ }^{244} \mathrm{Cm}$ & $-6 \% \pm 6 \%$ & $\begin{array}{c}\text { Decay heat, neutron } \\
\text { emitter, activity, burn } \\
\text { up-credit studies }\end{array}$ \\
\hline${ }^{154} \mathrm{Eu}$ & $+6 \% \pm 2 \%$ & $\begin{array}{c}\text { Burn up-credit studies, } \\
\text { decay heat }\end{array}$ \\
\hline${ }^{137} \mathrm{Cs}$ & $-4 \% \pm 2 \%$ & Decay heat \\
\hline
\end{tabular}

The DARWIN2.3 experimental validation work points out some isotopes for which the depleted concentration calculation can be improved; all the more some of them play an important role in the fuel cycle management (fuel inventory, decay heat, radiotoxicity...). Table 1 displays some examples of nuclides for which the calculation could be improved; and illustrates the results of the experimental validation for these nuclides.

The DARWIN2.3 calculation biases and uncertainties are defined with the $\mathrm{C} / \mathrm{E}$ values provided by the experimental validation. The uncertainties associated with $\mathrm{C} / \mathrm{E}$ values gather those due to the irradiation history modelling, those due to the burn up determination, and those due to the experimental uncertainties.

Some other isotopes such as ${ }^{14} \mathrm{C}$, have no available experimental validation, and the accuracy associated with the calculation of their concentration can be provided by a simplified propagation of a priori nuclear data covariance, provided that the latter are available.

Developing and improving the performances of a neutronic package relies on the VVUQ process, where VVUQ stands for Verification, Validation and Uncertainties Quantification. This method will be presented in the second part of this paper and a focus will be made on the need to strengthen the numerical validation of the DARWIN2.3 package. The work plan to improve DARWIN2.3 calculations is deduced from the VVUQ process and is presented in part 3. Investigations regarding first elements of modelling biases quantification will be handled in the fourth part. A conclusion and perspectives for this work will be presented in parts 5 and 6 .

\section{The VVUQ process}

The VVUQ approach is a rigorous method used to assess a simulation package and continuously improve its performances.

It comprises the three following steps.

- Verification: aims at verifying that the numerical resolution of neutronic solvers and the programming of each solver are correct, in order to avoid any regression when developing them.

- Validation: this step can be declined in two subphases.

- The numerical validation consists in comparing the results given by the package against the results given by a reference calculation, here it is with TRIPOLI4@ [10]. This allows a quantification of the biases induced by the modelling approximations in the resolution method of the calculation scheme tested. Given the fact that the package has to be as accurate as possible, it is necessary to reduce and quantify these biases.

- The experimental validation consists in comparing the results given by the package against experimental values. This step provides the $\mathrm{C} / \mathrm{E}$ values previously discussed.

- Uncertainty Quantification: the $(\mathrm{C} / \mathrm{E}) \pm \delta$ E obtained are transposed to the application domain of the package. If no experimental validation result is available, nuclear data uncertainties are propagated and the impact on the calculation of physical parameters is quantified.

\section{Work plan}

A work plan for the improvement of the DARWIN2.3 calculations has been established. It relies on identifying the potential ways of improving it. These opportunities are determined through the quantification and the analysis of biases and uncertainties engendered by the deterministic methods used in DARWIN2.3 calculations on the one hand and by nuclear data on the other hand. This quantification is carried out via numerical and experimental validation methodologies from the VVUQ process. The three axis of the work plan are presented in the following paragraphs.

The recent coupling of TRIPOLI4® stochastic transport code with the MENDEL deterministic depletion solver allows performing depletion calculation with TRIPOLI4R. Even though this process is not considered as a reference procedure for depletion calculation, the benchmarking of TRIPOLI4 $($ and DARWIN2.3 has been investigated to provide first elements of modelling biases quantification. Early results are presented in the following part.

Regarding the experimental validation, the feasibility of an experimental program in the MINERVE reactor at Cadarache is currently investigated. This program is called CARMINA for CARbon Measurement for Induced Neutron Activation. Its goal is to validate the ${ }^{14} \mathrm{C}$ buildup via ${ }^{17} \mathrm{O}(\mathrm{n}, \alpha)$ and ${ }^{14} \mathrm{~N}(\mathrm{n}, \mathrm{p})$ reactions, which are the major ways of formation in PWR UOX/MOX fuel and cladding.

A third approach to improve the DARWIN2.3 package calculations is to work on nuclear data, analyze it and re-estimate nuclear data by assimilation of integral experiment results. For instance, ${ }^{137} \mathrm{Cs}$ is systematically underestimated in PWR UOX fuel depletion calculations with DRAINW2.3 [1]. Assimilating this tendency could overcome this underestimation by reducing nuclear data uncertainties.

\section{TRIPOLI4.10 depletion calculations}

\subsection{Principle of the coupling}

To compute the concentrations without using a $100 \%$ Monte-Carlo sampling which would require a tremendous amount of resources, a coupling has been developed between two codes: TRIPOLI4®, which solves the 
Boltzmann transport equation thanks to a Monte-Carlobased method, and the MENDEL deterministic depletion solver.

A depletion calculation consists in a succession of transport and depletion phases, meaning that the irradiation time is divided in several steps. It is important to note that the uncertainty provided with first transport calculation is not propagated through the depletion step. In order to estimate more accurately the calculation uncertainty, several independent simulations are performed, and the distribution of the computed concentration values is studied. More details on the sources of uncertainty

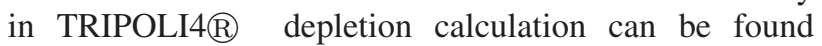
in $[11,12]$.

\subsection{Benchmarking details}

The geometry studied is a $3.7 \%$-enriched UOX PWR fuel cell, discretized in four parts to take into account the spatial heterogeneity of the flux. It is irradiated up to $60 \mathrm{GWd} / \mathrm{t}$, with a constant power value of $40 \mathrm{~W} / \mathrm{g}$ of heavy nuclides.

The comparison to our reference TRIPOLI4R calculation is not carried out with DARWIN2.3 but with APOLLO2. Since the TRIPOLI4® filiation chain is an optimized one to take into account the major reactivity contributors, it is compulsory to use APOLLO2 to make a consistent comparison since the DARWIN2.3 filiation chain is exhaustive. This means that a later goal will be to modify the filiation chains to take into account all the isotopes that are relevant for our study.

Therefore, a benchmark between an APOLLO2 calculation with the CYCLE2008-PWR scheme (to be consistent with DARWIN2.3 calculations) and a TRIPOLI4R calculation has been led.

\subsection{Early results}

Table 2 summarizes the results obtained for some nuclides of interest for the fuel cycle, classified by decreasing abundance at $60 \mathrm{GWd} / \mathrm{t}$.


is associated to the dispersion of the concentration values obtained with 64 independent replicas.

The discrepancies on the nuclides presented are below $1 \%$. The values can be compared with table 1: one can


low compared to the $\mathrm{C} / \mathrm{E}$ values. Thus, the major part of the discrepancies presented in Table 1 should be induced by nuclear data uncertainty.

It is likely that the self-shielding approximations and the flux solver used are responsible for the discrepancies given in Table 2. Further studies will be led to investigate both possibilities, respectively by performing a depletion calculation with a refined energy mesh (to dispense with self-shielding models) and by using a more accurate flux solver, based on the Method of characteristics (MOC).

\section{Conclusion}

This paper exposes the guidelines for the improvement of the calculation of nuclides of interest for the fuel cycle with the DARWIN2.3 package. A work plan has been established that can be split in three parts:
Table 2. Discrepancies between APOLLO2 and TRIPOLI4® depletion calculations for some important nuclides for the fuel cycle.

\begin{tabular}{|c|c|c|}
\hline Nuclide & $\begin{array}{c}\left(\mathrm{C}_{\mathrm{AP2}}-\mathbf{C}_{\mathrm{T} 4}\right) / \mathbf{C}_{\mathrm{T} 4} \\
(\text { in \%) }\end{array}$ & $\begin{array}{c}\text { TRIPOLI4® standard } \\
\text { deviation (in \%) }\end{array}$ \\
\hline${ }^{244} \mathrm{Cm}$ & -0.3 & 0.03 \\
\hline${ }^{154} \mathrm{Eu}$ & 0.5 & 0.02 \\
\hline${ }^{137} \mathrm{Cs}$ & 0.9 & 0.01 \\
\hline
\end{tabular}

- quantification of modelling biases using the numerical validation methodology, for which early results have been presented,

- computation of $\mathrm{C} / \mathrm{E}$ values using the experimental validation methodology, and

- nuclear data improvement.

The modelling biases quantification is tackled here to estimate its contribution on the $\mathrm{C} / \mathrm{E}$ values taken from the experimental validation report of the DARWIN2.3 package. Normally, one would expect these modelling biases to be low, since the CYCLE2008 calculation scheme is derived from the APOLLO2 SHEM-MOC scheme, which has been extensively validated. The results obtained here with a benchmark of APOLLO2 and TRIPOLI4R show interesting tendencies, since the discrepancies observed are rather low. Further work will focus on the quantification of the approximations made in APOLLO2 when using self-shielding models and a Pijbased flux solver.

\section{Perspectives}

The next step of the study will tackle the interpretation

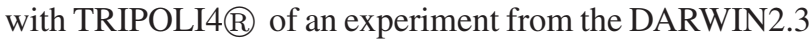
qualification report experimental database. This will allow comparing DARWIN2.3 against TRIPOLI4R and therefore having an accurate estimation of the modelling biases since the geometry, the irradiation history, etc. compared would be the same. A work on filiation chains will be done to have an even more accurate consistency between both calculations, wishing to confirm that the major part of the $\mathrm{C} / \mathrm{E}$ values is gathered by nuclear data.

In addition to this, and in order to extend the experimental validation of the DARWIN2.3 package, the CARMINA experimental program feasibility study is ongoing. It will aim at providing information for the ${ }^{14} \mathrm{C}$ concentration calculation.

More generally, it would be interesting to extend the experimental validation of the DARWIN2.3 package for nuclides of interest for the fuel cycle, like long-life fission products or activation products for instance. Very short (around the minute) and intermediate cooling time measurement of decay heat should also be investigated to provide reliable data for the experimental validation of decay heat calculation.

The third axis of this work plan will be to identify the potential way of improving nuclear data involved in the buildup of relevant isotopes. On a long-term period, the improvement of important nuclear data will be carried out and the global effect on the fuel inventory calculation quantified. 
We would like to thank our colleagues from the CEA who helped us for the implementation of the depletion calculation in TRIPOLI4®, especially E. Brun and Y. Pénéliau.

\section{References}

[1] L. San-Felice, R. Eschbach, P. Bourdot, Nucl. Tech. 184, 217-232 (2013)

[2] A. Santamarina et al., JEFF Report 22, OECD-NEA Data Bank

[3] R. Sanchez et al., Nucl. Eng. and Tech. 42(5), 474499 (2010)

[4] J.M. Ruggieri et al., Eranos2.1: The International Code System for GEN-IV Fast Reactor Analysis, Proc. Int. Conf. ICAPP (2006)

[5] A. Tsilanizara et al., DARWIN: an Evolution Code System for a Large Range of Applications, Proc. Int. Conf. ICRS-9 (1999)
[6] A. Santamarina, D. Bernard et al., APOLLO2.8: A validated code package for PWR neutronics calculations, Proc. Int. Conf. ANFM (2009)

[7] N. Hfaiedh, A. Santamarina, Determination of the Optimized SHEM mesh for Neutron Transport Calculation, Proc. Int. Conf. M\&C (2009)

[8] J.F. Lebrat et al., Analysis of the TRAPU and DOUBLON irradiations in PHENIX for the experimental validation of the DARWIN package for fast reactors, Proc. Int. Conf. GLOBAL (2015)

[9] J.C. Jaboulet, S. Bourganel, Nucl. Tech. 177, 73-82 (2011)

[10] E. Brun et al., Annals of Nucl. Eng. 82, 151-160 (2015)

[11] E. Dumonteil, C.M. Diop, Nucl. Sci. and Eng. 167, 165-170 (2011)

[12] E. Brun et al., Nucl. Sci. and Tech. 2, 879-885 (2011) 